\title{
Somatismen als Problem der dänischen und deutschen Lexikographie
}

Farø, Ken Joensen

Published in:

Symposium on Lexicography X. Proceedings of the Tenth International Symposium on Lexicography May 4-6 2000 at the University of Copenhagen

Publication date:

2002

Document version

Også kaldet Forlagets PDF

Document license:

Andet

Citation for published version (APA):

Farø, K. J. (2002). Somatismen als Problem der dänischen und deutschen Lexikographie. In H. Gottlieb, J. E. Mogensen, \& A. Zettersten (Eds.), Symposium on Lexicography X. Proceedings of the Tenth International Symposium on Lexicography May 4-6 2000 at the University of Copenhagen (pp. 107-124). Max Niemeyer Verlag. 


\section{Symposium on Lexicography X}

Proceedings of the Tenth International

Symposium on Lexicography May 4-6, 2000

at the University of Copenhagen

Edited by

Henrik Gottlieb, Jens Erik Mogensen

and Arne Zettersten

\section{Sonderdruck}

aus Lexicographica. Series Maior, Band 109 ISBN 3-484-39109-X

Max Niemeyer Verlag

Tübingen 2002

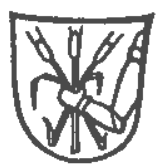


Table of Contents

Introduction

English.

German

Acknowledgements XVII

Norman Blake

Wolfgang Viereck

Tove Bjorneset

Jane Bottomley

Ulrich Busse

Timothy Colleman

Janet DeCesaris \& Victória Alsina

Bernhard Diensberg

Ken Farø

Henrik Gottlieb

Rolf Hesse

Lars Holm

Jean Hudson
Towards a Dictionary of Shakespeare's Informal English ........1

The Atlas Linguarum Europae

and its Insights into the Cultural History of Europe

The Dictionary Project NORDLEXIN-N

From Print to Disc: Creating the Electronic Version of the

Cambridge International Dictionary of English (CIDE).

Lexicography as a Sign of the Times:

A Study in Socio-Lexicography.

The Contragram Verb Valency Dictionary of Dutch, French and English

The Representation of Figurative Senses in Learner's Dictionaries .

Old French Loanwords of Germanic Origin

Borrowed into English.

Somatismen als Problem der dänischen und deutschen Lexikographie

Four Germanic Dictionaries of Anglicisms:

When Definitions Speak Louder than Words

Grammatische Schwierigkeiten bei der zweisprachigen Lexikographie

35 Questions and Answers about Editing

Jesper Swedberg's Swensk Ordabok.

Categorizing Chaos: Text Types in CANCODE .163 
Olga Karpova \&

Svetlana Manik

Ksenija Leban

Anatoly Liberman

Sándor Martsa

Geart van der Meer

Tadamasa Nishimura

Vilja Oja

Kurt Opitz

Yoshiaki Otani

Gunnar Persson

Vìra Schmiedtová \&

Barbara Schmiedtová

Whodzimierz Sobkowiak

Andrejs Veisbergs

Hideki Watanabe

Arne Zettersten \&

Hanne Lauridsen
Public Political Vocabulary: Model of a Dictionary .173

Towards a Slovene-English False-Friend Dictionary 185

The Length and Breadth of an Entry in an Etymological Dictionary.

Homonymy vs Polysemy: Conversion in English .211

Metaphors: How Do Dictionaries Scramble out of this Morass of Meaning?

Japanese Learners' Problems in Using

English-Japanese Dictionaries

Some Semantic Problems in the Translation of Colour Terms

The Dictionary of Connotations: A Viable Proposition?

Who Uses English-Japanese Dictionaries and When? Their Bidirectional Working

From 'Spinning Woman' to 'Old Maid' to What?

On the Sense Development of Spinster.

The Colour Spectrum in Language: The Case of Czech.

Cognitive Concepts, New Idioms and Lexical Meanings......285

On the Phonetics of Trans- in EFL Dictionaries

Euphemisms in General Monolingual Dictionaries

A Thesaurus of Old English Revisited

From Projection to Reception -

On the Process of Bilingual Dictionary Making. .325 


\section{Ken Farg}

Somatismen als Problem der dänischen und deutschen Lexikographie

\section{Einleitung}

"In der Phraseologie bezeichnet man herkömmlich alte Phraseologismen, die einen Körperteil als Komponente enthalten, als Somatismen", so Burger (1998:88). Das Phänomen ist auch unter teilweise alltagssprachlichen Bezeichnungen wie "Körper-Phraseologismus"" bekannt, wie z.B. bei Braun/Krallmann (1990:74). Die Kategorie umfasst Idiome wie $j m d n$. auf den Arm nehmen und Auge um Auge, Zahn um Zahn. Somatismen in diesem weiten Sinn bereiten der Lexikographie sicherlich nur insofern Schwierigkeiten, als sie überhaupt zur Kategorie der Idiomatik gehören. Auf das Problem der Idiomatik in der Lexikographie haben in den letzten 30-40 Jahren schon zahlreiche Forscher aufmerksam gemacht - speziell zum Thema Idiomatik in der bilingualen Lexikographie mit Dänisch und Deutsch (Farø 2000b). ' Über Idiomatik generell soll deshalb dieser Beitrag nicht handeln. Stattdessen soll die idiomatische Subkategorie der So-matismen besprochen werden, und zwar unter einem anderem Blickwinkel, als dies bisher geschehen ist.

Meine Haupthese ist, dass es sich für die Lexikographie lohnen würde, eine neue idiomatische Kategorie einzuführen, und zwar eine Kategorie, die ich Somatismen im engeren Sinn (S.i.e.S.) nenne. Das Ziel des Beitrags ist zu zeigen, wie diese bisher scheinbar unreflektierte Kategorie der Lexikographie Schwierigkeiten bereitet, sowie nicht zuletzt wie man diese Probleme lösen kann. Dabei ist die Perspektive auf die dänische und deutsche Lexikographie beschränkt, und zwar aus dem Skopus eines dänischen Mutter-sprachlers. Methodisch ist die Untersuchung empirisch ausgerichtet, weshalb die Wörterbücher vor allem auf der Grundlage von Korpusuntersuchungen ${ }^{2}$ uberpruft werden.

Der Beitrag gestaltet sich so, dass zunächst der Begriff Somatismus im engeren Sinn definiert wird. Gleichzeitig wird motiviert, warum diese Kategorie ein idiomatisches "Sonderproblem" darstellt. Im nächsten Schritt wird untersucht, wie die einzelnen Wörterbücher mit den Problemen umgehen, die diese lexikalische Kategorie der Lexikographie berejtet. Abschließend werden verschiedene Lösungsvorschläge für die dargestellten Probleme gegeben.

In der Germanistik v.a. Wissemann (1961), Burger (1983), Petermann (1983), ScholzeStubenrecht (1988), Schemann (1989), Schemann (1991), Burger (1992), Burger (1998:168-192).

2 Wichtige Einführungen in die Korpuslinguistik sind: Sinclair (1997), Mcenery/Wilson (1997), Kennedy (1998) og Biber et al. (1998). 


\section{Grundlage, Definition und Empirie}

\subsection{Grundlage}

Die Inspirationsquelle dieser Untersuchung waren nicht die spärlichen und m.E. nicht besonders ergiebigen Auseinandersetzungen der phraseologischen Forschung mit dem Begriff Somatismus. Sondern sie bestand zunächst in Frustrationen, die bei meiner eigenen, usuellen Wörterbuch-benutzung (siehe Wiegand 1998) entstanden sind. Außerdem wurden Burgers $(1976,1982)$ Arbeiten über die sogenannten "Kinegramme" exzerpiert.

Burgers Kinegramm-Begriff hat gewisse Affinitäten zu den hier introduzierten S.i.e.S., doch die Unterschiede sind eher auffallend. Zu den wesentlichsten Unterschieden gehört, dass Burgers Kinegramme sowohl poly- als auch monolexikalische Lexik umfassen, d.h. auch Lexeme wie lächeln, blinzeln u.ä., und dass der Begriff "Kinegramm"' impliziert, dass nur von solchen Lexemen die Rede ist, die eine Bewegung bezeichnen (vgl. griech. kinein 'bewegen' (Kluge 1989:370)).

Der in dieser Arbeit introduzierte Somatismus-Begriff beschränkt sich nicht auf Körperbewegungen, denn dies würde Syntagmen wie rote Ohren haben und jmdm. steht der Schweiß auf der Stirn ausschließen, die logisch zur Kategorie der S.i.e.S. gehören (vgl. unten).

Vor allem aber wird bei Burger das Problem der lexikographischen Kodifizierung von Kinegrammen nicht besprochen.

\subsection{Definition}

Folgende Auswahl an polylexikalischen Syntagmen:

die Stirn runzeln - rynke panden; rote Ohren bekommen/haben - fä/have rode orer; die Zähne zusammenbeißen - bide tcenderne sammen; sich die Haare raufen - rive sig $i$ häret; sich an den Kopf fassen-tage sig til hovedet; den Kopf schütteln - ryste på hovedet; krumme taer; sich an die Brust schlagen - slå sig for brystet; die Hände über dem Kopf zusammenschlagen- $\emptyset$

hat eine Reihe von Merkmalen gemeinsam:

1) Erstens sind sie Idiome i.e.S., d.h. lexikalisierte metaphorische polylexikalische Lexeme (vgl. Farø 2000a). ${ }^{4}$

2) Zweitens bestehen sie oberflächensemantisch (vgl, ibid.) ausschließlich aus somatischen Sememen.

3) Und drittens handelt es sich um solche Syntagmen, die nicht nur theoretisch, sondern auch real - d.h. empirisch nachweisbar - polysem sind. Sie werden sowohl idiomatisch, als auch litteral, d.h. kompositionell (vgl. Pinkal 1991), verwendet. ${ }^{\text {s }}$

Der sich wiederum auf den eher volkskundlich ausgerichteten Arbeiten RoHRIGs (z.B. 1967) stützt.

In dieser Arbeit, die eine empirisch basierte Konfrontation des danischen und deutschen idiomatischen Inventars durchführt, wird eine positiv definierte Idion-Kategorie vorgelegt. 
Wenn man die Somatismen i.e.S. von den Somatismen i.w.S. abgrenzt, dann müssen die beiden letzten Merkmale herangezogen werden. Somit sind mit der Faust auf den Tisch schlagen und etwas ist jmdm. ein Dorn im Auge, die von der Forschung (z.B. Lundh (1992) und Braun/Krallmann (1990)) als "Somatismen"' bezeichnet werden, keine Somatismen im engeren Sinn.- Denn ersterer enthält ein nicht-somatisches Lexem, Tisch, und der andere ist nicht polysem im engeren Sinn."

Dies gilt für die große Mehrheit der Somatismen, sowie für die Idiome allgemein: Ihre sogenannte "doppelte Lesart" (vgl. Burger 1998:59ff.), die von manchen Darstellungen der Idiomatik generell nachgesagt wird, ist oft ziemlich theoretisch. Denn selbst wenn ein foreign speaker ein Idiom wie $j m d m$. auf der Nase herumtanzen nicht unmittelbar verstehen würde, würde er sicherlich zu dem Schluss kommen, dass das Idiom nicht litteral interpretiert werden soll und deshalb nicht unkritisch in $L_{2}$ übertragen werden kann, und zwar aufgrund eines Analogieschlusses von seinen muttersprachlichen Idiom-Kenntnissen.'

Deshalb sind die Somatismen im weiteren Sinn, anders als die S.i.e.S., keine besonders problematische Lexemkategorie für die Lexikographie.

\subsubsection{Empirische Beispiele für die Polysemie der Somatismen i.e.S.}

Ein wichtiges Merkmal der S.i.e.S. ist also ihre Polysemie, und dass diese nicht bloß theoretisch, sondern in der Tat empirisch verifizierbar ist. Unten seien einige Beispiele für S.i.e.S. angeführt, deren sowohl idiomatische als auch litterale Realisierung anhand von Korpusbelegen nachgewiesen werden kann:

\section{1) die Stirn runzeln}

a) litteral

"Sensoren messen den elektrischen Hautwiderstand, den Blutdruck oder die Herzschlagfrequenz. Bewegungsmelder registrieren, wenn der Proband die Stirn runzelt, Kameras halten seinen Gesichtsausdruck fest" (DIE ZEIT 26.02.98, Auf dem Jahrmarkt der Forschung)

b) idiomatisch

"er erzăhlt Witze, bei denen sein Chef die Stirn runzeln und die Beförderungschancen neu überdenken warde" (STERN 05.11.87:32)

\section{2) den Kopf schutteln}

a) litteral

"Kein kuscheliger Ort. Den Namen eintragen, das Alter, und dann sch ittelt er den Kopf, nein, er sei nicht im Methadonprogramm" (DIE ZEIT 19.11.98, Auf Messers Schneide)

b) idiomatisch

"Was immer das Motiv der BBC-Spitze sein mochte, ob Angst vor dem einfluBreichen Minister oder die edle Absicht, seine Intimsphäre vor weiteren Übergriffen zu schützen - man setzte sich in

Burgers (1998:13) Einwände gegen diesen Begriff - ich habe seine Orthographie übernommen halte ich für nicht stichhaltig. Denn "wörtlich" ist eine gemeinsprachliche Bezeichnung, deren Verwendungsbereich breiter ist, als Burger annimmt - genauso wie "buchstäblich". Aus diesem Grund ist "litteral" ein geeigneterer fachsprachlicher Terminus.

Eine litterale Lesart von etwas ist jmdm. ein Dorn im Auge wirde die semantische Logik verletzen. Ob die Idiomatik eine universale Kategorie ist, ist m.W. ungeklärt. Zumindest scheinen aber alle indoeuropäischen Sprachen uber idiomatische Inventare zu verfügen. 
die Nesseln. BBC-Mitarbeiter sind wütend, die Öffentlichkeit schüttelt den Kopf' (DIE ZEIT 05.11 .98$, Piep)

3) rote Ohren bekommen

a) litteral

"Skifahren auf griechische Art ist ohnehin mit dem alpinen Pendant schwerlich zu vergleichen, obwohl es für rote Ohren und blaue Nasen allemal reicht" (DIE ZEIT 13.02.98, Wedeln bei den Göttern)

b) idiomatisch

"Das war kein Ruhmesjahr für sie: Sachverstăndige und viele Prognostiker der Forschungsinstitute haben die Konjunktur 1994 so schlecht vorausgeschätzt, daß sie noch jetzt rote Ohren haben mußten"' (DIE ZEIT 30.12.94, Aufschwung ohne Masse)

\subsubsection{Exkurs: Analysenprobleme und "Idiomatizitätsindikatoren",}

Wenn man potenzielle S.i.e.S. empirisch untersucht, d.h. in authentischem Text, so sind die beiden Realisierungsarten nicht immer so săuberlich voneinander zu trennen, wie es bei den drei obigen Beispielen der Fall war. Manchmal lässt sich nicht eindeutig feststellen, ob das Syntagma im jeweiligen Kontext idiomatisch oder litteral dekodiert werden soll, vgl. z.B.:

"Recht verdutzt war ein Zecher, der sich morgens um 7.30 die Augen rieb und sich allein in einem zugeschlossenen Lokal der Innenstadt befand" (MK 16.01.96, In der Kneipe eingeschlossen)

Das Syntagma sich die Augen reiben kann in diesem Text teils als ein rein extralinguistisches Morgenritual interpretiert werden, teils als ein idiomatischer Ausdruck fur die Überraschung des "'Zecher"'s, als er herausfindet, dass er in der Kneipe ein-geschlossen ist. Im letzteren Fall hat er sich kaum wirklich die Augen gerieben. Man kann den Text aber auch als eine bewusst zweideutige Formulierung auffassen, die beide Les-arten gleichzeitig realisiert, aber dies kann eben nicht eindeutig entschieden werden. Der Text ist ambig.

Es besteht aber nicht selten die Möglichkeit, dass man sich an etwas, was man "Idiomatizitäts-Indikatoren" nennen könnte, orientieren kann. Das sind hypothetisierende, futurisierende, potenzialisierende und generalisierende Elemente wie dürfte, würden, mögen, werden, man, mancher, jeder und immer. Solche Elemente kollokieren häufig mit S.i.e.S., die idiomatisch realisiert sind, wie z.B:

a) "Doch einige Fans dürften sich verdutzt die Augen reiben, wenn die 24jährige als lispelndes, ungemein blondes Dummchen auf der Leinwand erscheint" (MK) (potenzialisierender Idiomatizitätsindikator)

b) "mein Hausarzt (rümpft) immer die Nase, wenn jch Leberwurst... Doch das ist eine andere Geschichte" (MK) (generalisierender Idiomatizitatsindikator)

Hält man daran fest, dass S.i.e.S. polysem sind, dann muss die Funktion der Idiomatizitătsindikatoren sein, den ambigen Text zu monosemieren (vgl. Fleischer 1982:37). Denn die Idiomatizitätsindikatoren bewirken, dass das Syntagma in der konkreten Distribution ziemlich eindeutig als ein Idiom interpretiert werden muss. 


\subsection{Zusammenfassung vom Abschnitt 2}

Diese empirischen Belege machen deutlich, dass Somatismen i.e.S. in der Sprachwirklichkeit sowohl idiomatisch als auch litteral realisjert werden. Sie haben prinzipiell Koprâsenz von Idiomatizităt und Litteralitat (vgl. Wotjak 1986:186). Und dadurch unterscheiden sie sich von den Somatismen i.w.S.

Ein S.i.e.S. wird hier also als ein Idiom definiert, dessen Konstituenten-Semantik ausschließlich körperbezogen ist, und das idiomatisch und litteral kodiert ist, was empirisch nachweisbar ist.

\section{Somatismen i.e.S. kontrastiv}

Für einen Dänen sind die S.i.e.S. den Kopf schütteln, die Stirn runzeln und rote Ohren haben nicht besonders auffällig. Denn auch ihre dänischen Äquivalente sind polysem. Aus diesem Grund scheint die Frage berechtigt, ob diese lexikalische Kategorie überhaupt kontrastiv relevant ist? Denn wenn die Somatismen eine "Verbalisierung körperlichen Verhaltens" (vgl. Burger et al. 1982:57) darstellen, dann müsste man doch annehmen, dass es sich um Universalien handelt? Und müssen sie dann überhaupt von der bilingualen Lexikographie behandelt werden?

Dieser Einwand wăre unberechtigt, denn S.i.e.S. sind zwar möglicherweise als Kategorie eine universelle Erscheinung, doch im Einzelnen können kontrastive Unterschiede beobachtet werden:

\subsection{Deutsch vs. Dänisch}

Das Deutsche verfugt z.B. über den Somatismus i.e.S. die Hände über dem Kopf zusammenschlagen. Wie die Definition erfordert, kann es sowohl litteral als auch idiomatisch verwendet werden, obwohl das Syntagma sicherlich in idiomatischer Realisierung am häufigsten vorkommt." Es hat im letzten Fall eine Semantik, die man mit 'sehr erstaunt, überrascht, entsetzt sein' (Wahrig 2000) beschreiben kann. Betrachtet man dagegen das Dänische, so wird man feststellen, dass eine rein konstituentenbasierte Übersetzung von die Hände über dem Kopf zusammenschlagen ins Dänische ein Syntagma ergibt, das zwar sinnvoll ist, das aber dennoch keinen lexikalisierten semantischen Inhalt hat.

\footnotetext{
Korpusuntersuchungen (ZK) ergeben, dass von 8 Syntagmen die Hände über dem Kopf zusammenschlagen 5 eindeutig idiomatisch sind, während der Rest ambig ist.
} 


\section{Dănisch vs. Deutsch}

Kehren wir nun die Optik (Kromann 1995:118) um und machen das Dănische zum Ausgangspunkt, so kann das gleiche Phänomen anhand des Somatismus slå ud med armene beobachtet werden, das auf Deutsch etwa mit *die Arme herausschlagen wiedergegeben werden kann. Außer seiner litteralen Semantik hat das Syntagma auf Dănisch eine Reihe verschiedener lexikalisierter Bedeutungen als Idiom, darunter z.B. 'nicht zuruckhaltend sein", vgl:

"Reuter er styringsfreak. Der hvor jeg er god til at sıă ud med armene, sørger han benhärdt for, at der er dækning", beklager Preisler (BA)

"Men jeg har ingen formel for, hvordan man nærmer sig det poetiske. Poesien er en sjalden og meget sky fugl, hvis man kæfter for meget op og slår ud med armene, er den for længst fløjet" (BA)

Im Deutschen findet man offensichtlich nicht dieses Syntagma, weder als Idiom noch als litterale Kollokation. Es scheint, dass es sich um ein dänisches lexikalisches Schibboleth (vgl. Bußmann 1990:666) handelt.

Man könnte weitere Beispiele hervorheben, doch es soll hier kein voliständiges dänischdeutsches Inventar an somatischen Null-Äquivalenzen geliefert werden. Dagegen soll exemplarisch gezeigt werden, dass auch bei stark motivierten (siehe Burger 1998) Idiomen wie den polysemen Somatismen kein naives 1:1-Verhăltnis auf bilingualer Ebene gelten kann.

Die funktionale Äquivalenz und formale Parallelităt zwischen einigen dänischen und deutschen polysemen Somatismen, sowohl auf litteraler als auch auf idiomatischer Ebene, darf nicht dazu führen, dass sie von der Lexikographie als eine kontrastiv unproblematische Kategorie betrachtet werden. Polyseme Somatismen sind, mit einer Formulierung von Kromann/Kjær (1995:2), 'interlingual unvorhersagbar'"

\section{Somatismen i.e.S. in der dänischen und deutschen Lexikographie}

Wir wollen jetzt zur lexikographischen Behandlung der polysemen Somatismen übergehen. Die polysemen Somatismen sind eine in mehreren Hinsichten homogene Kategorie. Deshalb scheint es berechtigt davon auszugehen, dass in der Lexikographie standardisierte Prinzipien für ihre Behandlung gelten. Im Folgenden soll eine Reihe deutscher und dănischer Wörterbücher im Hinblick auf diesen Punkt untersucht werden. Zunächst soll aber der Frage nachgegangen werden, inwieweit diese lexikalische Kategorie uberhaupt von der Lexikographie berücksichtigt wird. Wir fragen damit nach dem Anteil der polysemen Somatismen an der Lemmaselektion der Wörterbücher.

"Siehe auch Rader (1998) und Becker-Christensen (1999). 


\subsection{Quantitativ: Zur Selektion von S.i.e.S. in der Lexikographie}

Fleischer (1982:19) kritisiert Phraseolexika wie Röhrig (1991 [1973]), weil hier die idiomatischen Kinegramme, und, kann man hinzufügen, auch die S.i.e.S., ausgeklammert werden. Er bemerkt, dass die Argumentation, diese Syntagmen hätten sich nicht weit genug von der ursprunglichen Bedeutung entfernt, "fur die moderne Phraseologieforschung nicht akzeptabel"' sei. Es könne nicht die Aufgabe eines Wörterbuchs sein, auch nicht die eines idiomatischen, "das Schwergewicht auf die kulturgeschichtlich und volkskundlich 'interessanten" " (Fleischer, ibid.) Idiome zu legen, wăhrend andere Idiome aus nicht nachvollziehbaren Gründen unberücksichtigt bleiben.

Von dieser Kritik ausgehend kann man fragen, inwieweit die dänische und deutsche Lexikographie die polyseme somatische Lexik berücksichtigt. Dies ist zugleich ein guter Ausgangspunkt für weitere Detailfragen zum Thema Somatismen in der Lexikographie (siehe 4.2.).

Zur Beantwortung dieser Frage wurden jeweils für Dänisch und Deutsch etwa 20 S.i.e.S. zusammengestellt um feststellen zu können, inwieweit diese lexikalische Kategorie von den Wörterbüchern kodifiziert ist.

Es handelt sich um folgende Wörterbicher: (Dänisch): Politikens Nudansk (= NU ${ }^{10}$ ), Danskordbogen (Systime) (= SY), Danske Talemåder (= DA); (Dänisch-Deutsch): DanskTysk (Gyldendal) (= GY $\mathrm{Y}_{\mathrm{da}-\mathrm{ty}}$ ), Dansk-Tysk Ordbog (Munksgaard) (= MU), Gads Large Dansk-Tysk (GAda-ty), Talemåder i dansk (= TA); (Deutsch-Dänisch): Tysk-Dansk (Gyldendal) (GY $\mathrm{G}_{\mathrm{ty}-\mathrm{da}}$ ), Gads Large Tysk-Dansk (GA $\mathrm{G}_{\mathrm{ty}-\mathrm{da}}$ ); (Deutsch): Duden Deutsches Universalwörterbuch (= DUW), Wahrig Deutsches Wörterbuch (WAHR), Langenscheidts Großwörterbuch. Deutsch als Fremdsprache (LA); Duden 11: Redewendungen (DU11) und schließlich Wörterbuch Deutsch als Fremdsprache (de Gruyter) (GRUY).

Will man einen Überblick darüber erhalten, welche Wörterbücher welche Somatismen enthalten, kann man die Ergebnisse der Suchfragen in einer Matrix darstellen. Zwei solcher Matrizen, jeweils mit Dänisch und Deutsch als Ausgangssprache, sind im Folgenden aufgeführt:

Alle Wörterbücher in dieser Untersuchung werden aus Platzgründen durch ein Signum wie dieses reprăsentiert. 
a) Dänisch

\begin{tabular}{|c|c|c|c|c|c|c|c|c|}
\hline Dänischer S.i.e.S. & $\begin{array}{l}\text { GY } \\
\text { da-ty }\end{array}$ & $\begin{array}{l}\mathrm{GA} \\
\text { da-ty }\end{array}$ & $\mathrm{MU}$ & $\overline{\mathrm{TA}}$ & SY & DA & NU & $\begin{array}{l}\text { Deutsches } \\
\text { Äquivalent }\end{array}$ \\
\hline 1. knibe sig i armen & - & - & - & - & + & - & + & $\begin{array}{l}\text { sich in den Arm } \\
\text { kneifen }\end{array}$ \\
\hline $\begin{array}{l}\text { 2. lægge armene over } \\
\text { kors }\end{array}$ & + & + & - & + & - & - & - & $\begin{array}{l}\text { die Arme kreuzen } \\
\text { (?) }\end{array}$ \\
\hline 3. siå ud med armene & - & - & + & - & - & + & + & $\varnothing$ \\
\hline $\begin{array}{l}\text { 4. stramme balderne/ } \\
\text { klemme balderne } \\
\text { sammen }\end{array}$ & + & - & + & + & + & + & + & $\begin{array}{l}\text { den Arsch } \\
\text { zusammenkneifen } \\
\text { (?) }\end{array}$ \\
\hline 5. ryste på hovedet & + & + & + & + & - & - & + & den Kopf schütteln \\
\hline 6. tage sig til hovedet & + & + & + & + & + & - & - & $\begin{array}{l}\text { sich an den Kopf } \\
\text { fassen }\end{array}$ \\
\hline 7. vride hænder & + & - & + & - & - & - & + & die Hände ringen \\
\hline $\begin{array}{l}\text { 8. ryste på } \\
\text { hæxnderne/hănden }\end{array}$ & + & - & + & - & + & + & + & $\begin{array}{l}\text { jmdm. zittern/zittert } \\
\text { die Hände/Hand / } \\
\text { jmds.... }\end{array}$ \\
\hline $\begin{array}{l}\text { 9. lagge hænderne i } \\
\text { skødet }\end{array}$ & + & - & + & + & - & + & + & $\begin{array}{l}\text { die Hände in den } \\
\text { Schoß legen }\end{array}$ \\
\hline 10. slå sig på lărene & - & - & + & + & - & - & - & $\begin{array}{l}\text { sich auf die } \\
\text { Schenkel schlagen }\end{array}$ \\
\hline 11. ăbne munden & - & $\overline{-}$ & + & + & - & - & + & $\begin{array}{l}\text { den Mund } \\
\text { aufmachen }\end{array}$ \\
\hline 12. rynke på næsen & - & + & + & + & + & + & + & die Nase rümpfen \\
\hline 13. rynke panden. & + & + & + & + & + & - & + & die Stimn runzeln \\
\hline 14. løfte pegefingeren & - & - & - & $\rightarrow$ & - & + & + & $\begin{array}{l}\text { den Zeigefinger } \\
\text { heben }\end{array}$ \\
\hline 15. trække på skuldrene & + & + & + & + & - & + & + & \begin{tabular}{|l|} 
die/mit den \\
Schultern zucken
\end{tabular} \\
\hline 16. have sved på panden & - & - & + & $\overline{-}$ & + & - & - & $\begin{array}{l}\text { jmdm. steht der } \\
\text { SchweiB auf der } \\
\text { Stirn }\end{array}$ \\
\hline $\begin{array}{l}\text { 17. vende tommel- } \\
\text { fingeren nedad }\end{array}$ & - & - & - & - & + & + & + & \begin{tabular}{|l|} 
den Daumen nach \\
unten halten \\
\end{tabular} \\
\hline 18. trille tommelfingre & + & - & 7 & + & + & + & + & $\begin{array}{l}\text { Daumen/Däumchen } \\
\text { drehen }\end{array}$ \\
\hline 19. få/have røde ører & - & - & - & - & + & - & + & $\begin{array}{l}\text { rote Ohren } \\
\text { bekommen/haben }\end{array}$ \\
\hline
\end{tabular}


b) Deutsch

\begin{tabular}{|c|c|c|c|c|c|c|c|c|}
\hline Deutscher S.i.e.S. & $\begin{array}{c}G Y \\
\text { ty-da }\end{array}$ & $\begin{array}{l}\mathrm{GA} \\
\mathrm{da}-\mathrm{ty}\end{array}$ & DUW & $\begin{array}{c}\text { GRU } \\
Y\end{array}$ & LA & $\begin{array}{c}\text { Dul } \\
1\end{array}$ & $\begin{array}{c}\text { WAH } \\
\mathrm{R} \\
\end{array}$ & $\begin{array}{l}\text { Dänisches } \\
\text { Äquivalent }\end{array}$ \\
\hline 1. sich die Augen reiben & - & - & + & + & - & - & + & gnide sig i øjnene \\
\hline $\begin{array}{l}\text { 2. sich an die Brust } \\
\text { schlagen }\end{array}$ & + & - & + & - & - & + & - & $\varnothing$ \\
\hline $\begin{array}{l}\text { 3. Daumen/Däumchen } \\
\text { drehen }\end{array}$ & + & + & + & + & + & + & + & trille tommelfingre \\
\hline $\begin{array}{l}\text { 4. den Daumen nach } \\
\text { unten halten }\end{array}$ & - & - & - & - & - & - & - & $\begin{array}{l}\text { vende tommelfinge- } \\
\text { ren nedad }\end{array}$ \\
\hline 5. kalte Füße bekommen & + & - & + & + & + & + & + & fă kolde fødder \\
\hline 6. sich die Haare raufen & + & + & + & - & + & + & + & rive sig i håret \\
\hline $\begin{array}{l}\text { 7. die Hände in den } \\
\text { Schoß legen }\end{array}$ & - & - & + & + & + & + & + & $\begin{array}{l}\text { lægge hænderne i } \\
\text { skødet }\end{array}$ \\
\hline 8. sich die Hände reiben & + & + & - & + & - & + & \pm & gnide sig i hænderne \\
\hline 9. die Hände ringen & + & - & + & - & - & - & + & vride hænder \\
\hline $\begin{array}{l}\text { 10. jmdm. zittern/zittert } \\
\text { die Hände/Hand / jmds... }\end{array}$ & - & - & + & + & + & - & + & $\begin{array}{l}\text { ryste på hænderne/ } \\
\text { hånden }\end{array}$ \\
\hline $\begin{array}{l}\text { 11. die Hände über dem } \\
\text { Kopf zusammenschlagen }\end{array}$ & + & - & + & + & + & + & + & $\varnothing$ \\
\hline $\begin{array}{l}\text { 12. sich an den Kopf } \\
\text { fassen }\end{array}$ & + & + & + & - & + & + & + & tage sig til hovedet \\
\hline 13. den Mund aufmachen & - & - & + & + & + & + & + & åbne munden \\
\hline 14. die Nase rümpfen & + & + & + & + & + & + & + & rynke næsen \\
\hline $\begin{array}{l}\text { 15. rote Ohren ha- } \\
\text { ben/bekommen }\end{array}$ & - & - & + & - & - & + & - & have/fă røde ører \\
\hline $\begin{array}{l}\text { 16. jmdm. steht der } \\
\text { Schweiß auf der Stirn }\end{array}$ & - & - & + & + & + & - & - & have sved på panden \\
\hline 17. die Stirn runzeln & - & - & + & + & + & - & + & rynke panden \\
\hline $\begin{array}{l}\text { 18. die Zähne } \\
\text { zusammenbeißen }\end{array}$ & + & - & + & + & + & + & + & $\begin{array}{l}\text { bide tændeme sam- } \\
\text { men }\end{array}$ \\
\hline $\begin{array}{l}19 . \text { den Zeigefinger } \\
\text { heben }\end{array}$ & - & - & - & + & + & - & - & løfte pegefingeren \\
\hline
\end{tabular}

Die Matrizen sind wie folgt zu lesen: In der äußersten linken Spalte stehen die untersuchten Somatismen, und in der obersten die Signa der Wörterbücher. Wenn ein Somatismus im Wörterbuch kodifiziert ist, wird dies mit einem Pluszeichen markiert, Nichtvorhandensein markiert ein Strich. Ganz rechts sind, wenn vorhanden, das jeweils deutsche oder dänische Äquivalent aufgeführt. Fragezeichen markieren nicht ganz sichere Äquivalente.

Aus den Matrizen geht hervor, dass die polysemen Somatismen vielfach nicht kodifiziert sind: Tatsächlich sind in etwa $40 \%$ der Fälle die jeweiligen Somatismen in weniger als der Hälfte der Wörterbücher aufgeführt. Es gibt sogar S.i.e.S., die in keinem der untersuchten Wörterbücher vorkommen, nämlich sich in den Arm kneifen und den Daumen nach unten halten. Dass beide Somatismen im Deutschen keine Exotismen, sondern durchaus lexi- 
kalisiert sind, kann anhand von "Korpora" wie Die Zeit auf CD-ROM 1995-1999 (ZK) und Frankfurter Allgemeine auf CD-ROM 1995-1997 (FK) nachgewiesen werden.

Interessant ist dabei, dass kein signifikanter Unterschied zwischen der gemeinsprachlichen Lexikographie und der Speziallexikographie (den drei Phaseolexika, TA, DA und DU11) festgestellt werden kann: Der durschnittliche Deckungsgrad dieser Idiom- wörterbücher liegt knapp unter dem Gesamtdurchschnitt von $57 \%$. Die Priorităt der Somatismen i.e.S. scheint also für beide Lexikographietypen gleich (niedrig) zu sein.

Wenn ein so relativ großer Teil dieser lexikalischen Kategorie in der mono- und bilingualen Lexikographie mit Dänisch und Deutsch nicht kodifiziert ist, so liegt dies sicherlich an ihrer Zwitterstellung zwischen einerseits den "'klassischen Idiomen" (vgl. Clausén 1994:48) wie das Zünglein an der Waage sein und jmdm. das Messer auf die Brust setzen und andererseits den Kollokationen. D.h. häufig vorkommenden Syntagmen ohne eine idiomatische Dimension, wie etwa sich das Haar waschen und zu Abend essen. (Zum Problem der Kollokationen: siehe z.B. Cop 1991 und Burger 1998:50ff.). Die S.i.e.S. haben an beiden Kategorien teil, und dies verursacht offensichtlich Unsicherheit beim Lexikographen.

Obwohl diese Syntagmen zur "Kernlexik" (Bergenholtz/Mugdan 1990:1618) der beiden Sprachen gehören, werden sie im Allgemeinen von der Lexikographie quantitativ nicht gebührend berlicksichtigt. Dass dieses Problem auch eine qualitative Komponente hat, daruber soll im Folgenden die Rede sein.

\subsection{Qualitativ: Identifikation und Markierung}

\subsubsection{Fehlende Identifikation als polysemes Syntagma}

a) Unten ist der Wortlaut aus einem Artikel eines deutsch-dänischen Wörterbuchs aufgeführt:

zusammenbeißen tr. bide sammen ( $\mathrm{fx}$ die Zähne) (GY $\mathrm{GY}_{\mathrm{ty}-\mathrm{da}}$ )

Auf den ersten Blick scheint dieser Artikel unproblematisch zu sein, doch der aufmerksame Benutzer wird wissen, dass das deutsche Ausgangssyntagma polysem ist: es kommt sowohl in einer idiomatischen als auch einer litteralen Variante vor. Doch auf welche dieser Varianten bezieht sich das Äquivalent? Vielleicht auf beide? Das Wörterbuch gibt keine Hinweise darauf.

Das Problem an dieser Stelle besteht also darin, dass das Syntagma nicht eindeutig identifiziert wird: als bloßes Idiom, als bloß litterale Kollokation, oder als polysemer Somatismus mit beiden Dimensionen, was hier am angemessensten scheint.

b) Ein Eintrag aus einem dänisch-deutschen Wörterbuch:

rynke panden die Stirn runzeln $\left(\mathrm{GA}_{\mathrm{da}-\mathrm{ty}}\right)$

Dass dieses Syntagma polysem ist, geht aus dem Wörterbucheintrag nicht hervor. Hiermit wird der Benutzer im Stich gelassen, denn wie soll er die spärliche Information über das 
Lemma interpretieren, die sich auf das Anführen eines Äquivalentes beschränkt? Für einen Benutzer, der etwa den folgenden Text ins Deutsche übersetzen muss:

Olsen pointerede, at han ikke var den eneste, der rynkede panden over redegørelsen (BA98),

sind die Informationen in diesem Worterbuch nicht viel wert. Denn er wird räsonieren, dass rynke panden im Ausgangstext idiomatisch benutzt wird, und als unsicherer Sprachproduzent weiß er nicht, ob auch das Äquivalent idiomatisch kodiert sein kann und ob es gegebenenfalls die gleiche Funktion hat wie das dänische Idiom.

Die fehlende Identifikation des Syntagmas als litteral oder idiomatisch scheint vor allem ein Problem der bilingualen Lexikographie zu sein. Dies hängt sicherlich damit zusammen, dass diese weniger explizit ist als die monolinguale, weil sie ja in erster Linie die Lemmata nicht beschreiben, sondern bloß Übersetzungsvorschläge liefern solt (Vgl. KROMANN et al. 1984).

\subsubsection{Einseitige Identifikation}

In der monolingualen Lexikographie (vgl. Geeraerts 1989 und Wiegand 1989) dagegen ăußern sich die Schwierigkeiten anders. Denn hier muss der Lexikograph explizit das Lemma beschreiben. Aus diesem Grund wird jedesmal deutlich, wie das Syntagma seitens des Lexikographen interpretiert worden ist, ob als Kollokation, Idiom oder eben als beides auf einmal, d.h. als polysemer Somatismus.

\subsubsection{Einseitige Monosemierung zugunsten der litteralen Dimension}

In den untersuchten Wörterbüchern werden die polysemen Somatismen of einseitig als litterale Kollokationen beschrieben, obwohl sie im Sprachgebrauch auch idiomatisch verwendet werden.

a) Z.B. findet man in einem monolingualen deutschen Wörterbuch unter dem Lemma reiben diese Angabe:

sich etwas (einen Körperteil) die Hand od. Finger mit Druck darauf hin und her bewegen 2 sich die Augen (WAHR)

Das Syntagma ist ein Somatismus i.e.S., weil es im Deutschen sowohl litteral als auch idiomatisch realisiert wird. Zur idiomatischen Realisierungsvariante, siehe z.B.:

Doch einige Fans dürften sich verdutzt die Augen reiben, wenn die 24jährige als lispelndes, ungemein blondes Dummchen auf der Leinwand erscheint (MK)

Die Bedeutungserklärung sieht über die idiomatische Dimension des Syntagmas hinweg. Für den Benutzer ohne sichere Deutschkenntnisse, vor allem furr diejenigen foreign learners, in deren Sprachen sich die Augen reiben keine idiomatische Realisierungsvariante hat, ist der Artikel wenig hilfreich, wenn es um die Interpretation von Texten wie oben geht. 
b) Ein weiteres Beispiel für Einseitigkeit zugunsten der litteralen Realisierungsvariante der polysemen Somatismen in einem deutschen monolingualen Wörterbuch liefert der folgende Eintrag:

ringen 3. (geh.) a) (die Hände) aus Verzweiflung, Angst o.ä. falten, ineinander verschränkt gegeneinander pressen $u$. so in drehender Bewegung aneinander reiben: weinend, flehend die/seine Hănde r.

Es steht außer Frage, dass hier die litterale Variante des Syntagmas beschrieben wird. Genauso sicher ist aber, dass die idiomatische Realisierung im Sprachusus mindestens genauso häufig ist." Diese Dimension wird aber von diesem Worterbuch nicht berluck-sichtigt.

\subsubsection{Einseitige Monosemierung zugunsten der idiomatischen Dimension}

Das Gegenteil ist aber auch weit verbreitet, nämlich dass der polyseme Somatismus einseitig zugunsten der idiomatischen Dimension identifiziert wird.

a) Z.B. wird in einem dänischen monolingualen Wörterbuch der Somatismus løfte pegefingeren, was im Deutschen den Zeigefinger heben entspricht, auschließlich als Idiom beschrieben:

lefte pegefingeren påpege noget moralsk betænkeligt finansministeren havde den loftede pegefinger fremme (NU)

Dass es sich außerdem um eine Kollokation handelt, wird verschwiegen.

b) Das Syntagma die Hände über dem Kopf zusammenschlagen wird in einem deutschen Wörterbuch auf folgende Weise beschrieben:

gespr; sehr erstaunt od. erschrocken sein (LA),

also als Idiom. Doch damit wird uber die potenzielle litterale Dimension hinweggesehen, die ja keineswegs ungewöhnlich ist.

Offensichtlich bildet diese einseitige Monosemierung kein signifikantes Muster, etwa indem das eine Wörterbuch die polysemen Somatismen nur idjomatisch darstellt, während sie vom anderen ausschließlich litteral interpretiert werden: Vielmehr entscheidet das einzelne Wörterbuch von Fall zu Fall jeweils anders, und zwar keineswegs konsequent. ${ }^{12}$

Zusammenfassend kann also die Tendenz beobachtet werden, dass die polysemen Somatismen in der bilingualen Lexikographie nicht monosemiert und in der monolingualen Lexikographie einseitig monosemiert werden und zwar relativ unsystematisch. Es handelt sich um kein Randphänomen, sondern die Tendenz ist so verbreitet, dass sie bei etwa $90 \%$ der

1) Vgl. ZK 1999.

12 Abgesehen von SY, das im Prinzip nur Idiome bringt und keine Kollokationen (vgl. die Einleitung) - obwohl dieses Prinzip nicht konsequent eingehalten wird. Dass die Idiome nicht erklärt werden, halte ich für eine Schwäche des Wörterbuchs. 
kodifizierten polysemen Somatismen in den gemeinsprachlichen Wörterbüchern festgestelit werden kann."

5. Drei Musterartikel: Vorschläge zur Behandlung der S.i.e.S. in der Lexikographie

Oben wurden Beispiele für problematische Kodifizierungen von polysemen Somatismen in der deutschen und dänischen Lexikographie gezeigt. Es wurde festgestellt, dass es sich um ein generelles lexikographisches Problem handelt. Nichtsdestoweniger gibt es einige Ausnahmen. Im Folgenden werden diese herangezogen um herauszufinden, wie eine optimale Artikelstruktur der polysemen somatischen Lexik aussehen könnte:

\section{1. sich die Hände reiben}

In einem deutschen einsprachigen Wörterbuch wird das Syntagma sich die Hände reiben folgendermaßen beschrieben:

sich die Hände reiben (um sie zu erwärmen, vor Vergnügen od., aus Schadenfreude); <fig.> Schadenfreude empfinden, schadenfroh sein (WAHR)

Die Struktur des Artikels ist zweiteilig, indem das Lemma in zwei verschiedenen Sektionen erläutert wird, zunächst einer litteralen, dann einer iđiomatischen, die explizit mit " figürlich" markiert ist. Das Wörterbuch interpretiert das Syntagma offensichtlich als polysem, weshalb es monosemiert werden muss. Der Artikel macht also den Benutzer darauf aufmerksam, dass das Syntagma synchronisch sowohl einen rein somatischen Vorgang bezeichnet, und dass dieses Syntagma außerdem als Metapher lexikalisiert ist, mit der Semantik 'schadenfroh sein'. Die Reihenfolge ist logisch, weil die idiomatische Variante als sekundäre Nomination (vgl. Fleischer 1982:166ff.) in der Form einer Ab-leitung der ersteren betrachtet werden muss.

Diese Methode ist optimal, weil beide Realisierungsvarianten kodifiziert und bedeutungsexpliziert werden, was das Wörterbuch nicht zuletzt für den foreign learner nlitzlich macht.

\section{2. tage sig til hovedet}

Munksgaard Dansk-Tysk (= MU) ist ein bilinguales Wörterbuch für Benutzer mit Dänisch als $\mathrm{L}_{1 .}$. Das Worterbuch kann also zu einem gewissen Grad die muttersprachliche Kompetenz des Benutzers ausnutzen. Gleichzeitig muss ein Produktionswörterbuch im Prinzip

13. Selbstverständlich wäre es sinnlos, die drei Phraseolexika in diesen Teil der Untersuchung einzabeziehen, weil diese ja prinzipiell nur die idiomatische Dimension der Syntagmen einbeziehen. 
nichts als Äquivalente liefern. Deshalb muss die Beschreibung des S.i.e.S. hier nicht so umfangreich sein wie in einem monolingualem Wörterbuch wie WAHR, wo der Benutzer teils adäquate Bedeutungserklărungen erwartet, und wo teils das Benutzersegment heterogener ist als die von MU. ${ }^{14}$ Deshalb kann sich MU mit diesem Eintrag begnügen:

tage sig til hovedet (også fig.) - sich an den Kopf fassen/greifen

Weitere Informationen hat der Benutzer im Prinzip nicht nötig: teils wird ein adäquates Äquivalent gegeben, teils muss der Benutzer die Markierung (ogsi fig.) als einen Hinweis darauf verstehen, dass das deutsche Äquivalent sowohl litteral als auch idiomatisch benutzt werden kann. ${ }^{15}$

Merkwürdigerweise kann jedoch auch festgestellt werden, dass das Wörterbuch unter dem naheliegendsten Nachschlagekonstituenten, hoved, wo das Syntagma auch behandelt wird ein Verfahren benutzt, das dem Benutzer nicht die nötige Hilfe gibt. ${ }^{16}$. Denn dieser kann im Prinzip nicht wissen, ob der Eintrag:

tage sig til hovedet - sich an den Kopf greifen

ein litterales Äquivalent, idiomatisches Äquivalent oder eine Kombination von beidem ist. Der Lexikograph kann nicht voraussetzen, dass der Benutzer aus dieser Äquivalensrelation einen Analogieschluss macht, im Sinne von: wenn es sich um Äquivalente handelt, dann sowohl in wörtlichem als auch in übertragenem Gebrauch. Wie wir in 3. gesehen haben, ist dies ja nicht immer der Fall.

\section{3. trille tommelfingre (= Daumen/Däumchen drehen)}

Ein letztes Beispiel dafur, wie man das Problem bewältigen kann, liefert ein dänisches monolinguales Wörterbuch. Seine Methode besteht darin, dass zwei getrennte Subartikel für das Formativ konstruiert werden:

- trille tommelfingre flette begge hænders fingre sammen og dreje tommelfingrene rundt om hinanden

- trille tommelfingre udtryk for at man keder sig

Obwohl der Unterschied zwischen diesen beiden Syntagmen nicht explizit als einer zwischen litteraler und idiomatischer Realisierung beschrieben wird, so sind die beiden verschiedenen Varianten deutlich hervorgehoben und voneinenander getrennt, mit einer $\mathrm{Be}$ schreibung der jeweiligen Semantik.

14 WAHR wird in hohem Maße auch von foreign speakers gekauft (vgl. Vorwort zur Neuausgabe 1994).

Is Obwohl eine Platzierung der Markierung hinter dem Äquivalent sicherlich zweckmäßiger gewesen wäre. Denn die Markierung soll sich wohl nicht auf das dänische Syntagma beziehen? Was die $L_{i}$ betrifft, muss der Lexikograph die muttersprachliche Kompetenz des Benutzers ausnutzen können. Was in Faø (2000a) unter dem Begriff Polyrepräsentation behandelt wird. 
Der Nachteil dieser Methode ist aber, dass sie verhältnismaßßig viel Platz in Anspruch nimmt. $\mathrm{Zu}$ bemerken ist außerdem, dass die zweite Bedeutung missverstanden werden kann, weil "udtryk for" ("Ausdruck für") polysem ist. Gleichzeitig ist es nicht ganz unwesentlich hervorzuheben, dass die Methode, zwei isolierte Subartikel zu konstruieren, in NU keineswegs konsequent angewandt wird: In der Tat wird sie bei keinem anderen der 19 polysemen Somatismen benutzt, die untersucht worden sind. Auch hier dominiert die einseitige Identifikation (zugunsten entweder der litteralen oder der idiomatischen Dimension), wie sie in 5.2.2. beschrieben wurde.

Diese drei Artikel stellen jeweils fur die mono- und bilinguale Lexikographie Musterbeispiele dafür dar, wie das Problem S.i.e.S. gelöst werden kann, ohne dass zu große Anforderungen an die sprachliche Kompetenz des Benutzers oder den Umfang des Wörterbuchs gestellt werden. Die Methoden an sich sind keineswegs innovativ, da sie in der Lexikographie ja bereits benutzt werden, nämlich wenn ein Lexem in einer oder in beiden Sprachen sowohl in litteralem als auch in idiomatischem Gebrauch vorkommt. ${ }^{17}$

Man kann diese Methoden unter dem Begriff explizite Polysemie zusammenfassen. Alle drei Musterartikel gehen explizit mit der Polysemie dieser Syntagmen um und liefern, auf jeweils unterschiedliche Weise, vorbildliche lexikographische Lösungen für dieses idiomatische Sonderproblem.

Nicht die Lösungsvorschläge selber, sondern die Erkenntnis, dass diese polysemen Syntagmen ein besonderes lexikographisches Problem darstellen, ist in diesem Zusammenhang das Wesentliche.

\section{Zusammenfassung}

Ich habe in diesem Beitrag die lexikographische Behandlung einer idiomatischen Subkategorie untersucht, nämlich die Somatismen im engeren Sinn (S.i.e.S.) oder polysemen Somatismen. Diese Kategorie besteht aus denjenigen polylexikalischen Syntagmen, deren Semantik ausschließlich körperbezogen ist, und die außerdem polysem sind, d.h. sie kommen als Syntagmen sowohl in litteraler als auch in idiomatischer Realisierung vor.

Wenn diese lexikalische Kategorie introduziert wird, so liegt es daran, dass sie teils eine wichtige Ergänzung zur Klassifikation der Idiomatik ist und teils weil nachgewiesen werden kann, dass sie, zumindest in der dänischen und deutschen Lexikographie, häufig mangelhaft und unzweckmäßig behandelt wird.

Somit kann festgestellt werden, dass die S.i.e.S. in der bilinguaten Lexikographie meistens nicht monosemiert werden: Ihre Polysemie wird häufig ignoriert. In der monolingualen Lexikographie dagegen werden sie zwar monosemiert, doch meistens in Form einer Vereinseitigung. Dabei kommt einseitige Monosemierung sowohl zugunsten der litteralen, als auch zugunsten der idiomatischen Dimension vor.

${ }_{17}$ Ein nicht-somatisches Beispiel wäre Alarm schlagen, das aber genauso wenig wie die S.i.e.S. in den hier untersuchten Wörterbüchern zuffiedenstellend behandelt wird. 
Die S.i.e.S. befinden sich auf der Nahtstelle zwischen Kollokationen und Idiomatik. Und dies bringt offensichtlich oft den monolingualen Lexikographen dazu, sie nach einem Entweder-Oder-Prinzip zu behandeln, wo es m.E. am zweckmăßigsten wäre, diese Kategorie lexikographisch nach einem Sowohl-Als-Auch-Prinzip zu beschreiben, d.h. beide Realisierungsvarianten müssten explizit im Artikel auftreten.

Für die praktische Lexikographie ist die explizite Idiomklassifikation kaum zweckmăßig. Somit würde es den Wert des Wörterbuchs kaum steigern, wenn man den S.i.e.S. eine besondere Markierung geben würde, vielleicht eher im Gegenteil. ${ }^{18}$

Stattdessen lohnt sich eine pragmatische Methode. Ein solcher Pragmatismus konzentriert sich um den Polysemieaspekt an den S.i.e.S. Für eine gelungene Wörterbuchbenutzung ist es eine Voraussetzung, dass ambige Lexeme jeder Art monosemiert werden. In diesem Punkt unterscheiden sich die polysemen Somatismen nicht von der sonstigen polysemen Idiomatik - oder gar von der polysemen monolexikalischen Lexik.

Das Besondere an den S.i.e.S. ist jedoch, dass ihre Polysemie ein konstitutives Merkmal ist. Dies macht die S.i.e.S. zum nutzlichen Prüfstein dafür, ob ein Wörterbuch mit polysemen Lemmata systematisch und benutzerfreundlich umgeht.

Über Klassifikationen von Phraseologie hat Fleischer (1982:29) gesagt: "'Der Wert der Klassifikationsversuche darf [...] nicht überschătzt werden [...] Die Klassifikation ist nicht Selbstzweck'. Klassifikationen haben demnach nur einen Wert, wenn sie in konkreten Problemen ihren Ausgangspunkt nehmen, die mit den existierenden Kategorien nicht zufriedenstellend gelöst werden können. Hoffentlich ist es diesem Beitrag gelungen, die Richtigkeit dieser Aussage zu illustrieren.

\section{Literatur}

a) Nachschlagewerke

Bußmann, Hadumod (1990): Lexikon der Sprachwissenschaft. Stuttgart: Kröner.

DU11 = Drosdoswki, Gunther/Scholze-Stubenrecht, Werner (1992): DUDEN Redewendungen und sprichwörterlichen Redensarten. Wörterbuch der deutschen Idiomatik. Mannheim: Dudenverlag.

DUW = Drosdowki, Günther et al. (1996): DUDEN Deutsches Universalwörterbuch. 3. Auflage. Mannheim: Dudenverlag.

GAda-ty = Hansen, Carsten (1993): Gads Tysk Large: tysk-dansk/dansk-tysk ordbog. København Gad.

18 Genauso wie man generell vermeiden sollte, in Wörterbuchern, vor allem in bilingualen, mit verschiedenen phraseologischen Subklassen zu operieren. Somit ist das Vorhaben in Dittmer (1981) nicht nachvollziehbar, in einem dänisch-deutschen Wörterbuch zwischen Idiomen wie bide $i$ del sure ceble und bide $i$ grcesset unterscheiden zu wollen, derart, dass diese Idiome unterschiedlich markiert werden und an verschiedenen Stellen in der Mikrostruktur stehen sollen. Die Begründung sieht der Verfasser in der unterschjedlichen Motiviertheit der Idiome. Einerseits ist aber diese angebliche Transparenz des ersten Idioms ein völlig subjektives Kriterium, andererseits scheint diese Aufteilung für ein solches Wörterbuch überhaupt zwecklos. 
GRUY = Kempcke, Günther et al. (2000): De Gruyter Wörterbuch Deutsch als Fremdsprache. Berlin: de Gruyter.

GYda-ty = Bork, Egon (1999b): Dansk-Tysk Ordbog. 11. Ausgabe. København: Gyldendal

GYty-da $=$ Bork, Egon (1999a): Tysk-Dansk ordbog. 14. Ausgabe. Kobenhavn: Gyldendal.

LA = Götz, Dieter et al. (1993): Langenscheidts Großwörterbuch Deutsch als Fremdsprache. Berlin/Zürich: Langenscheidt.

MU = Bergstrøm-Nielsen, Henrik et al. (1996): Dansk-tysk ordbog. 2. Auflage. København: Munksgaard.

NU = Becker-Christensen, Christian (1999): Politikens Nudansk Ordbog med etymologi. København: Politiken.

SY = Bergenholtz, Herning (1999): Danskordbogen. Danmark: Systime.

WAHR = Wahrig, Gerhard (2000): Deutsches Wörterbuch. Köln: Bertelsmann.

b) Artikel und Monographien

Bergenholtz, Henning/Mugdan, Joachim (1990): Formen und Probleme der Datenerhebung II: synchronische und gegenwartsbezogene Wörterbilcher. In: Hausmann et al. (1990):1611-1625.

Biber, Douglas et al. (1998): Corpus Linguistics. Investigating language structure and use. Cambridge: Cambridge University press.

Braun, Peter/Krallmann, Dieter (1990): Inter-Phraseologismen in europäischen Sprachen. In: Braun, Peter/Schaeder, Burkhard/Volmert, Johannes (Hgg.) (1990): Internationalismen. Studien zur interlingualen Lexikologie und Lexikographie. Tübingen: Niemeyer. S. 74-86.

Burger, Harald (1976): "die achseln zucken". In: Wirkendes Wort, 26:5. S. 31 1-334.

Burger, Harald/Buhofer, Annelies/Sialm, Ambros (1982): Handbuch der Phraseologie. Berlin; de Gruyter.

Burger, Harald (1983): Phraseologie in den Worterbüchern des heutigen Deutsch. In: Germanistische Linguistik 1-4, 82. S. 13-66.

- (1989): Phraseologismen im allgemeinen einsprachigen Worterbuch. In: Hausmann et al. (1989):593-599.

- (1992): Phraseologie im Wörterbuch. In: Matesic, Josip (1992): Studia Phraseologica et alia. Festschrift für Josip Matesic zum 65. Geburtstag. München: Peter Lang. S. 33-51.

- (1998): Phraseologie. Eine Einführung am Beispiel des Deutschen. Berlin: Erich Schmidt Verlag.

Clausén, Ulla (1994): Idiom och variation. In: Garde, Anna/Jarvad, Pia (red.): Nordiske Studier Leksikografi II. Rapport fra konference om leksikografi i Norden 11.-14. maj 1993. Oslo: Nordisk Forening for Leksikografi. S. 47-52.

Cop, Margaret (1991): Collocations in the bilingual lexicography. In: Hausmann et al. (1991): 2775 2779 .

Dittmer, Arne (1981): Feste Syntagmen im dänisch-deutschen Worrterbuch. In: Kopenhagener Beiträge zur germanistischen Linguistik 17. S. 110-125.

Faro, Ken (2000a): Dansk-tysk kontrastiv idiomatik. En empirisk baseret konfrontation af det moderne danske og tyske idiominventar. København: Kobenhavns Universitet. (Magisterarbeit).

- (2000b): Idiomatik i moderne dansk-tysk leksikografi. In: Hermes 25, s. 171-202..

Fleischer, Wolfgang (1982): Phraseologie der deutschen Gegenwartssprache. Leipzig: Bibliographisches Institut.

Geeraerts, Dirk (1989): Principles of monolingual lexicography. In: Hausmann et al. (1989):287-296.

Hausmann, Franz Joseph/Reichmann, Oskar/Wiegand, Herbert Ernst/Zgusta, Ladislav (Hgg.) (1989/1990/1991): Wörterbücher. Ein internationales Handbuch der zeitgenössischen Forschung. Berlin/New York: de Gruyter.

Kennedy, Graeme (1998): An Introduction to Corpus Linguistics. London: Longman.

Kluge, Friedrich (1989): Etymologisches Wörterbuch der deutschen Sprache. Berlin/New York: de Gruyter

Kromann, Hans-Peder (1995): Von den Möglichkeiten einer kontrastiven Optik und Mikroskopie in der Lexikologie. In: Kromann/Kjær (1995):114-126. 
Kromann, Hans-Peder/Riiber, Theis/Rosbach, Poul (1984): Überlegungen zu Grundfragen der zweisprachigen Lexikographie. In: Germanistische Linguistik 3-6/84.

Kromann, Hans-Peder/Kjær, Anne Lise (1995): Von der Allgegenwart der Lexikologie. Kontrastive Lexikologie als Vorstufe zur zweisprachigen Lexikographie. Akten des internationalen Werkstattgesprächs zur kontrastiven Lexikologie 29.-30.1094 in Kopenhagen. Tubingen: Niemeyer.

Lundh, Karin (1992): Wie wird der Kaiser zum Papst? Zur etymologischen Bedeutung zweier Somatismen im Deutschen und Schwedischen. In: Der Gingkobaum 11. S. 42-49.

McEnery, Tony/Wilson, Andrew (1997) [1996]: Corpus Linguistics. Edinburgh: Edinburgh University Press.

Petermann, Jürgen (1983): Zur Erstellung ein- und zweisprachiger phraseologischer Wörterbücher: Prinzipien der formalen Gestaltung und der Einordnung von Phrasemen. In: Matesic (1983): Phraseologie und ihre Aufgaben. Beiträge zum I. Internationalen Phraseologie-Symposium vom 12. bis 14. Oktober 1981 in Mannheim. Heidelberg: Groos. S. 172-191.

Pinkal; Manfred (1991): Vagheit und Ambiguität. In: von Stechow/Wunderlich (1991): Semantik. Ein internationales Handbuch der zeitgenössichen Forschung. Berlin: de Gruyter. S. 250-269.

Røder, Allan (1998): Danske Talemåder. København: Gad.

Röhrig, Lutz (1967): Gebärde - Metapher-Parodie. Düsselforf: Schwann.

- (1991) [1973]: Das große deutsche Wörterbuch der sprichwörllichen Redensarten. Freiburg im Breisgau:Herder.

Schemann, Hans (1989): Das phraseologische Wörterbuch. In: Hausmann et al. (1989):1019-1032.

- (1991): Die Phraseologie im zweisprachigen Wörterbuch. In: Hausmann et al. (1989):2789-2794.

Scholze-Stubenrecht (1988): Phraseologismen im Wörterbuch. In: Das Wörterbuch: Artikel und Verweisstrukturen. Sprache der Gegenwart 74. S. 284-302.

Sinclair, John (1997) [1991]: Corpus, Concordance, Collocation. Oxford: Oxford University Press.

Wiegand, Herbert Ernst (1998): Wörterbuchforschung. Untersuchungen zur Wörterbuchbenutzung, zur Theorie, Geschichte, Kritik und Automatisierung der Lexikographie. 1. Teilbd: Berlin: de Gruyter.

- (1989): Der gegenwärtige Status der Lexikographie und ihr Verhältnis zu anderen Disziplinen. In: Hasumann et al. (1989): 246-280.

Wissemann, Heinz (1961): Das Wortgruppenlexem und seine lexikographische Erfassung. In: Indogermanische Forschungen LXVI. S. 225-258.

Wotjak, Gerd (1986): Zur Bedeutung ausgewăhlter verbaler Phraseologismen des Deutschen. In: Zeitschrift für Germanistik 2. S. 183-200.

\section{c) Korpora}

$\mathrm{BA}=$ Berlingske Avisdata (CD-ROM)

FK = Frankfurter Allgemeine Zeitung 1995-1997 (CD-ROM)

MK = Mannheimer Korpora (www.ids-mannheim.de)

ZK= Die Zeit 1995-1999 (CD-ROM) 